\title{
Nivel de conciencia de la actividad y creación de información en medios digitales. Un estudio de caso en estudiantes universitarios chilenos
}

\author{
Marisol P. Hernández-Orellana ${ }^{1}$, Adolfina Pérez-Garcias ${ }^{2}$ y Ángel G. Roco-Videla ${ }^{3,4 \star}$ \\ (1) Unidad de Desarrollo Corporativo, Informática Educativa, Universidad Autónoma de Chile, Galvarino Gallardo 1983, \\ Santiago-Chile (correo-e: marisol.hernandez@uautonoma.cl). \\ (2) Departamento de Pedagogía Aplicada y Psicología de Educación, Universidad de las Islas Baleares, Carretera de \\ Valldemossa, km 7.5, 07122 Palma, Illes Balears-España (correo-e: fina.perez@uib.es). \\ (3) Facultad de Ciencias de la Salud, Universidad Bernardo O'Higgins, General Gana 1702, Santiago-Chile \\ (correo-e: angelroco@postgrado.ubo.cl). \\ (4) Facultad de ingeniería, Departamento de Ingeniería Civil, Universidad Católica de la Santísima Concepción, Alonso \\ de Rivera 2850, Concepción-Chile (correo-e: aroco@ucsc.cl).
}

* Autor a quien debe ser dirigida la correspondencia

Recibido Nov. 30, 2020; Aceptado Ene. 28, 2021; Versión final Mar. 28, 2021, Publicado Ago. 2021

\begin{abstract}
Resumen
Los objetivos de esta investigación fueron establecer el grado de conciencia de la actividad en la red en un grupo de estudiantes universitarios y, además, determinar cómo dicho grado condiciona la creación de información. En este estudio descriptivo no experimental de corte transversal, basado en el paradigma cuantitativo, se encuestó a 509 estudiantes a quienes se les aplicó dos cuestionarios, uno para establecer el nivel de conciencia de la actividad en red, y otro para describir los hábitos de trabajo y aprendizaje para analizar el proceso de creación de información para la red. Se determinó que un $10.81 \%$ de los estudiantes tenía una alta conciencia de su actividad en red y que la producción de fotografías era el principal tipo de contenido compartido. Se concluyó que una menor conciencia de la actividad implicaba una menor producción de contenidos y una menor utilización de herramientas específicas para su generación.
\end{abstract}

Palabras clave: identidad digital; entorno personal; aprendizaje; conciencia; actividad; observador;

publicadores

\section{Awareness level of information activity and creation in digital media. A case study in Chilean university students}

\begin{abstract}
The objectives of this research study were to establish the degree of awareness of online activity in a group of university students and to determine how this degree conditions the creation of information. In this descriptive non-experimental cross-sectional study, based on a quantitative paradigm, 509 students were surveyed with two questionnaires. One to establish the level of awareness of online activity and the other to describe work and learning habits to examine the process of creating information online. The results showed that $10.81 \%$ of the students surveyed had high awareness of their online activity and that making pictures was the main type of shared content. In conclusion, the less awareness about online activity implied lesser content creation and less use of specific tools for its generation.
\end{abstract}

Keywords: digital identity; personal learning environments; activity awareness; lurkers; posters 


\section{INTRODUCCIÓN}

Hoy en día es factible procesar y compartir datos desde un computador de escritorio, notebook, tablet, smartphone, reloj inteligente e incluso desde la pantalla táctil de un refrigerador. Gracias al desarrollo de la domótica, prácticamente cualquier lugar de nuestro hogar es un punto de conexión que nos permite compartir y recibir información. Por tanto, es dable decir que la tecnología impacta continuamente a la sociedad, así como también el comportamiento y la forma en que las personas se relacionan y comunican entre sí; impulsando una mayor visibilidad gracias a modos de digitalización cada vez más complejos (Torres Kompen et al., 2019). Unido a la incorporación de los celulares inteligentes, transformados en herramientas integrales e indispensables, tanto para desarrollar la vida social y profesional como la académica; permitiendo una constante comunicación en medios digitales cuya presencia virtual se tornó omnipresente (Soomro et al., 2019).

En este escenario de alta participación tecnológica, las plataformas digitales brindan un oportunidad para promocionar y posicionar marcas y productos en el mundo, difundir ampliamente ideas sobre género y sexismo (Wiens et al., 2020), como también para visibilizar a las personas influyéndolas en función de los datos digitales personales que son explotados en este nuevo entorno social y político creado por las Tecnologías de la Información y Comunicación (TIC) (Lange, 2020). Todo lo cual facilita una omnipresencia en la Red que permite la constante generación de información, pero es aquí donde nos preguntamos, ¿qué tan conscientes somos de nuestra actividad en la Red y cómo esta conciencia afecta nuestra producción de información y, de paso, nuestra identidad?

En esta línea reflexiva Sibilia (2010), en su libro El Hombre Postorgánico, analiza conceptos como cuerpo, subjetividad y el biopoder, entrecruzando biología e informática, para exponer que el cuerpo humano se estaría volviendo obsoleto al reducirlo a un sistema de procesamiento de datos, cuyo anhelo e imperativo sería "el deseo de lograr una total compatibilidad con el tecnocosmos digital. Ello mediante la actualización tecnológica permanente" (Sibilia, 2010; p.13). Bauman, considerando la sentencia de Alain Touraine de que el ser humano como ser social ha muerto, va un paso más allá, planteando que los individuos ya no nacen con una identidad, por lo que aquello que les era consustancial se ha transformado en una tarea personal impostergable de la vida moderna (Bauman, 2015). Este proceso performativo de construcción de la identidad digital implica, necesariamente, una negociación en el espacio social de la Web mediante el lenguaje visual y la escritura, los cuales deben estar alineados, tanto los intereses y deseos del usuario como, asimismo, del entorno social preexistente en la comunidad virtual que lo acoge (Belli et al., 2014).

En el proceso de construcción de las identidades la persona se ve inmersa en un dilema, dado que la modernidad fragmenta, pero también une y unifica. Por ello, con la llegada de Internet y las redes sociales la actividad intragrupal (clave para la conformación de la identidad personal) se mantiene y la construcción personal (individual-social) adquiere relevancia y se amplía. Sin embargo, los rasgos macrosociales, es decir, la actividad intergrupal queda suprimida a su mínima (Çöteli, 2019). Por consiguiente, la identidad digital trae aparejada una dicotomía entre lo individual/grupal en su construcción (Belli et al., 2014). De allí que se hace más complejo diferenciar entre identidad y reputación online. Siendo la identidad el complejo conjunto de datos e información formado por todo lo que decimos y hacemos en Internet, así como también, por lo que los demás dicen que hacemos o decimos. En tanto, la reputación digital apunta a la interpretación que otros se hacen del individuo a partir de toda esta información; construyéndose socialmente basado en las interacciones de la contribución individual de ideas, información y consejos que emite y que los otros usuarios valoran (Brandtzaeg, 2020; Abdallah, 2018).

Para comprender de mejor manera cómo se construye y qué indicadores considera la identidad digital Georges (2012) crea un modelo teórico sin desconocer el carácter fluido, contextual, complejo y multifactorial que esta implica. Se establece que la identidad digital está compuesta de tres dimensiones: a) declarada, b) creada y c) calculada. La identidad declarada es la primera que entra en acción cuando el internauta registra su información personal relevante y específica exigida para dar existencia al ser online que interactuará en la Web. De modo que la identidad creada opera y se configura a partir del momento que la persona entra en acción en el ámbito virtual (Brandtzaeg, 2020), proporcionando información por medio del rastro de su actividad en el sistema como, asimismo, de las acciones sucesivas de interacción y actualización propias de un usuario con vida en la Web (Georges, 2012).

La última dimensión es la identidad calculada, que es la expresión final de las dos anteriores. En este caso, es el propio sistema el que genera automáticamente la información a partir de dos categorías: a) variables cualitativas que consideran información atributiva sobre la presencia y actividad actual del usuario; centrando la atención en indicadores relativos al estado de conexión como en línea/fuera de línea; disponible/no disponible; etc. y b) variables cuantitativas referidas al número de amigos, puntaje y rango en que es categorizada la persona acorde al sitio. Estos son datos significativos porque cuantifican la presencia, visibilidad y reputación del usuario y lo compara y clasifica en función de la cultura local. Por ende, el mensaje 
de aumento de amistad o de seguidores tiene un impacto inmediato en el individuo respecto de su jerarquía en el sistema (Georges, 2012).

De modo que, las huellas digitales que deja una persona al utilizar aquellas herramientas y servicios de la Web 4.0, que le son más accesibles y cómodas, le acompañarán de forma permanente (Abdallah, 2018; Kumar et al., 2018; Feher, 2019), afectando todas las dimensiones que configuran las identidades personales y grupales, conjuntamente con las respectivas reputaciones, sino también la arquitectura del entorno personal de aprendizaje, más conocido por su sigla en inglés PLE (Personal Learning Environments), (Torres Kompen et al., 2019; Dabbagh et al., 2020). El PLE se relaciona con la forma concreta de entender cómo se aprende, en qué contexto y con quiénes se aprende más que centrarnos en una herramienta en particular, por lo que un PLE implica todo aquel conjunto de herramientas, fuentes de información y servicios disponibles en la Web que permiten que las personas pueden acceder, construir, gestionar y compartir contenidos educativos para satisfacer sus necesidades habituales de aprendizaje personal, social y profesional (Dabbagh et al., 2020; Şahin et al., 2016).

El entorno personal de aprendizaje nace al alero de las investigaciones referidas al uso de la inteligencia artificial para la generación de entornos personales de aprendizaje (1977) y hacia las décadas de los 80 y 90 se avanza hacia el aprendizaje colaborativo asistido por computadoras. Finalmente, gracias a la difusión y exploración del uso de las TIC y las redes sociales en el enriquecimiento del entorno de aprendizaje virtual tradicional, en el año 2005, nos acercamos al concepto moderno (Dabbagh et al., 2020). Para esclarecer el sentido del constructo PLE se centró la mirada en los tres procesos cognitivos básicos que este facilita: leer, reflexionar e informar. Comprendiendo todas aquellas herramientas que permiten a las personas informarse, procesar dicha información y compartir sus miradas particulares relacionándose con otros (Dabbagh et al., 2020; Şahin et al., 2016; Torres Kompen et al., 2019).

De acuerdo con lo anterior, el PLE sitúa al usuario en el centro de toda la actividad, entregándole la libertad de decisión respecto desde donde: a) accederá a la información, b) modificará la información y c) se relacionará con otros (Şahin et al., 2016; Torres Kompen et al., 2019). Por ende, un PLE debiera estar constituido por, al menos, tres líneas de herramientas y estrategias: 1) las ligadas a la lectura comprendida por las fuentes de información (blogs, wikis, YouTube, repositorios, buscadores de bases de datos, etc.); 2) las de reflexión que considera los entornos o servicios para transformación información (wikis, Google Docs, aplicaciones para infografías, afiches, pósters, mapas conceptuales, etc.) y 3) las de relación que apuntan a las redes personales de aprendizaje o PLN, por su acrónimo en inglés, que varían de acuerdo con la finalidad, encontrándose las de establecimiento de relaciones por: i) interacción comunicativa con otras personas (Linkedln, Facebook); ii) aprendizaje por medio de objetos de información (YouTube, Slideshare, Vimeo, Pinterest, etc.); iii) compartir experiencias y recursos de aprendizaje de interés (Twitter) (Guodong et al., 2016; Şahin et al., 2016; Dabbagh et al., 2020).

Dentro de las ventajas distintivas del PLE se encuentran que son herramientas de acceso abierto, permitiendo la descarga gratuita, personalizables al ser gestionadas por el usuario, multihilo o multilateral, posibilitando la participación simultánea en interacción y cooperación cuya conveniencia y usabilidad están garantizadas sin restricciones de sesgo o edad. Además, la sociabilización se ve favorecida por los canales de comunicación escrita y visual que permiten al usuario conocer, crear, compartir y actuar autorreflexivamente. Por último, los PLE, al contar con amplias redes de usuarios, favorecen el predominio o adhesión a comunidades de interés.

Esta investigación tiene como objetivo describir y establecer la conciencia de la actividad de un grupo de estudiantes universitarios y establecer cómo el grado de dicha conciencia condiciona la creación información que comparten en la red.

\section{OTROS ANTECEDENTES}

Las estrategias personales en línea siguen decisiones mayoritariamente conscientes, cuyas huellas digitales son expresión de un modelo intercultural e intergeneracional que opera mediado por la interfaz de la computadora incidiendo en la construcción de la identidad digital (Feher, 2019; Georges, 2012). Asimismo, las múltiples herramientas de las tecnologías 3.0 y 4.0, conjuntamente con las plataformas de redes sociales, alientan a los estudiantes a extender sus PLE a PLN por lo que la socialización y colaboración, no solo es social, sino académica al interactuar con sus compañeros de clase y docentes generándose una simbiosis entre identidad digital y PLE (Basantes et al., 2017; Dabbagh et al., 2020).

El componente tecnológico y social que configura el PLE de cada estudiante le permite controlar y autogestionar su aprendizaje, así como también, contribuir a su proceso de socialización incidiendo, necesariamente, en la construcción de su identidad digital y reputación profesional (Şahin et al., 2016). De modo que, en la medida que conocemos la forma en que los jóvenes crean su identidad digital se avanza, 
conjuntamente, en la comprensión de cómo generan sus experiencias sociales y culturales y viceversa (Castañeda et al., 2012).

Por tanto, de la selección del conjunto de líneas de herramientas y estrategias que constituirán el PLE personal se desprenderá una serie de definiciones que cimentarán el camino tecnológico, social, pedagógico, ideológico, temático, profesional y personal del individuo (Rejón-Guardia et al., 2020). De modo que la autorregulación del aprendizaje individual apoyado con herramientas digitales puede dar cuenta de un usuario más o menos reflexivo (Guodong et al., 2016), un individuo con o sin una conciencia ecológica digital (si se saben o no conectados con el grupo y perciben dicha presencia reconocible en el entorno virtual), consumidor o productor de contenido (prosumidor), o bien, desde su participación como observadores (lurkers) 0 publicadores (posters) en la Web (Nguyen, 2020).

Existen múltiples variantes de usuarios, pero todos están conectados a una forma particular de aprender y configurar su PLE e identidad digital (Torres Kompen et al., 2019). Así, por ejemplo, los observadores son aquellos participantes en la Red que solo leen las publicaciones de otros y no hacen una contribución propia y, pese a lo que pudiese pensarse, son la contante en las comunidades virtuales. La explicación es simple, el individuo que nunca publica en la Red es aquel que ha perdido la confianza en su autoeficacia del conocimiento creyendo que no tiene nada qué aportar al resto o que no cuenta con una amplia gama de competencias digitales. No obstante, los observadores son necesarios porque entregan valor a la comunidad en línea, es decir, en las plataformas de comunicación y redes sociales coexisten personas cuyos mismos intereses y objetivos motivan a los publicadores a mantener su actividad aportando al conocimiento colectivo (Nguyen, 2020).

Este nuevo desarrollo identitario, compuesto por la hibridación entre el mundo presencial y el digital, formal e informal, público y privado conlleva un cambio en los procesos de enseñanza-aprendizaje y un desafío para las instituciones de formación superior. El profesional que egresa se insertará en un entorno dinámico con una fuerte influencia digital y tecnológica, que le exigirá ser digitalmente autónomo para actualizar sus conocimientos y competencias frente a las versátiles necesidades actuales y futuras (Basantes et al., 2017). Frente a este escenario, como docentes y diseñadores de aulas virtuales y plataformas educativas, parecemos ignorar esta valiosa información para crear buenas prácticas pedagógicas, educar en el autocuidado digital e integrar adecuadamente las TIC en lo presencial y virtual en las salas de clases.

De hecho, existen investigaciones relacionadas con la comprensión de la identidad profesional vinculada a las redes sociales (Brandtzaeg et al., 2020), con la representación de la identidad en las redes sociales y la privacidad digital en estudiantes europeos y asiáticos (Feher, 2019). Huang et al. (2018) en su trabajo nos señalan que las mujeres comparten más información en las redes ya que esto les favorece en la construcción de su identidad social personal y profesional abordando el impacto significativo en los intercambios producidos en función de una red digital profesional. A nivel de competencias digitales hay investigaciones que apuntan al alumno a través de su avatar en entornos de simulación 3D estableciendo una pauta de observación relativa a la apariencia del avatar dentro del entorno a nivel individual (Esteve-González et al., 2017) o enfocadas en la exploración de las percepciones y preocupaciones de los estudiantes españoles de educación superior sobre su identidad digital (Castañeda et al., 2012).

Por otra parte, las investigaciones socio-antropológicas mexicanas caminan hacia la visibilidad en espacios públicos y privados con los que se identifica y construye la identidad de los jóvenes en internet (Winocur, 2012). En tanto, el estudio griego sobre las redes sociales y su influencia en la construcción de la identidad juvenil se direcciona en cómo se podría caracterizar el ámbito de acción y comunicación de Facebook considerando la esfera pública, semipública o privada (Georgalou, 2016). Sin embargo, ninguna de estas investigaciones considera la identidad digital y el PLE como un todo único y completo, aplicado a un contexto latinoamericano.

\section{METODOLOGÍA}

Esta investigación se desarrolló dentro de la lógica del pragmatismo, vía alternativa al pospositivismo y constructivismo. Reforzando el pluralismo y la sinergia apuntando a un modelo de investigación flexible de tipo no experimental, descriptiva, de corte transversal, basada en el paradigma cuantitativo. La muestra no probabilística o dirigida, definida en función una población de tamaño infinito o desconocido abarcó un total de 509 sujetos voluntarios pertenecientes a 21 de las 55 universidades chilenas activas a la fecha, repartidos a su vez en 51 carreras. De acuerdo con el criterio de inclusión, consideró a todos los participantes que tenían 18 años o más (mayoría de edad en Chile) y cursaban una carrera en modalidad presencial diurna. Antes de contestar los instrumentos, todos los participantes recibieron una carta de consentimiento informado indicando los detalles de la investigación y de su participación. Considerándose los criterios de confidencialidad de la información establecidos en la declaración de Helsinki. 
Para la recolección de la información se utilizaron dos instrumentos, el primero de ellos correspondió a una encuesta con escala tipo Likert de 6 opciones que tiene como finalidad describir la identidad digital creada, declarada y calculada, siendo la conciencia de la actividad una de las subdimensiones de la identidad calculada. Este instrumento fue creado por el equipo de investigación y fue validado mediando el juicio de expertos utilizando la técnica de grupo nominal (Barroso Osuna et al., 2013), dicho grupo estuvo conformado por 12 doctores pertenecientes a universidades europeas y latinoamericanas, divididos en dos grupos: a) con publicaciones en identidad digital y b) con publicaciones en tecnología educativa en general. Para la validación psicométrica se determinó un tamaño muestral de 384 sujetos, de cuyos resultados se obtuvo un alfa de Cronbach de 0.872 para identidad digital declarada, 0.913 para la identidad digital creada y un 0.916 para la identidad digital calculada. El muestreo fue de tipo no probabilístico con una confianza del $95 \%$ y un error muestral del 5\%. El cuestionario "Identidad Digital" estaba compuesto por cuatro dimensiones. La primera dimensión nominada "datos personales" aborda una serie de datos sociodemográficos de contextualización.

La segunda dimensión denominada "conocimientos y actitudes ante la identidad digital" engloba un conjunto de indicadores que dan cuentan desde el manejo teórico de los constructos básicos, ligados a identidad y reputación digitales, así como también, de sus percepciones y preferencias relativas a estos. En tanto la tercera dimensión, "conectividad y uso de dispositivos", está direccionada a precisar el escenario tecnológico y técnico desde el cual acceden los estudiantes a la Web. Finalmente, la cuarta dimensión relativa a la "definición de identidad digital" que expresan y manifiestan los estudiantes se tomó como sustrato teórico el modelo planteado por la Dra. Fanny Georges (2012) sobre construcción de la identidad digital considerándose las llamadas identidad declarada, creada y calculada.

Los expertos en su conjunto se pronunciaron respecto de la claridad, coherencia y pertinencia de las preguntas que conformaban el instrumento, calificándolo de 1 a 4 siendo el 1 el no cumplimiento con el criterio y 4 el nivel más alto de cumplimiento. Asimismo, frente a cada pregunta se les dispuso un recuadro en caso de que requirieran emitir una evaluación cualitativa. A partir de los informes individuales de juicio, emitido por cada uno de los expertos, se elaboró una tabla de recomendaciones con las observaciones cualitativas y las frecuencias de los puntajes obtenidos. Como criterio para descartar una pregunta se debería cumplir dos requerimientos conjuntamente: a) Que la frecuencia obtenida fuera inferior a 0.75 . b) Que, al menos el 0.75 de las observaciones de los expertos del panel, señalen que debe ser omitida. De acuerdo con las observaciones del panel de expertos y los criterios de eliminación el cuestionario se vio afecto a la reformulación de redacción de los encabezados y de algunas preguntas, mientras otras se fusionaron.

El segundo instrumento utilizado fue el cuestionario sobre hábitos de trabajo y aprendizaje, el cual explora los entornos personales de aprendizaje (PLE) y que fue desarrollado por el equipo de investigación español liderado por Prendes-Espinosa et al. (2016). Este instrumento en su dimensión de "Gestión de la información" consta de una subdimensión denominada "Creación de la información", que fue la utilizada en esta investigación. Este instrumento ya validado por sus autores usando un panel de expertos, fue validado semántica y psicométricamente para ser aplicado en la población chilena. Se debió ajustar el término "colega", siendo reemplazado por "compañero" dado que la población objeto de estudio no corresponde a una fuerza trabajadora.

La validación estadística del instrumento desarrollado en España fue realizada en una población de 384 sujetos, según determinación de tamaño muestral para una proporción en una población de tamaño infinito con un nivel de confianza del $95 \%$ y un error muestral del 5\%, el muestreo fue no probabilístico. La aplicación de este instrumento siguió los mismos protocolos establecidos para el resto de esta investigación. El valor del alfa de Cronbach para la subdimensión de "Creación de la información" fue de 0.8403 cumpliendo con lo esperado para un instrumento descriptivo.

Se entenderá por conciencia de actividad al conocimiento o autopercepción consciente que se tiene respecto de la regularidad con que se realizan acciones o actividades en la Red. Para categorizar el nivel de "conciencia de la actividad" y poder así analizar la dimensión de "creación de la información" en función de dicha graduación, se consideró la estructura de construcción del instrumento de identidad digital que considera 6 niveles. Dicha encuesta usa una escala tipo Likert, donde todas las preguntas asociadas a la "conciencia de la actividad" estaban graduadas en forma ascendente en cuanto a la intensidad que declara el sujeto frente a cada afirmación. A cada nivel de la escala Likert se le asignó un puntaje acorde a su intensidad obteniendo la siguiente secuencia de puntaje: Siempre/Casi siempre(S/CS) $=6$ pts.; A menudo $(A M)=5$ pts.; A veces $(A V)=4$ pts.; Pocas veces $(P V)=3$ pts.; Casi nunca/Nunca $(C N / N)=2$ pts.; Nunca/No aplica $(U N / N A)=1$ pt.

Para generar una categorización del nivel de conciencia, se agruparon las respuestas de los participantes. Dado que la escala Likert utilizada tiene 6 niveles de intensidad, la agrupación más adecuada es la de tres categorías. Las intensidades más bajas que son 1 y 2 , se fusionaron para constituir el nivel "bajo de conciencia" que, dado el número de preguntas, permiten tener un puntaje cuyos valores fluctúan entre un mínimo de 7 y un máximo de 14. La "mediana conciencia" se constituyó por la fusión de las intensidades 3 y 
4, con un puntaje que fluctúa entre 15 y 21 puntos. Finalmente, la "alta conciencia" estará constituida por las intensidades 5 y 6 con un puntaje que fluctúa entre 22 y 42 puntos. A partir de esta categorización se realizará el análisis de los datos relacionados con la creación de información y el tipo de información creada según lo declarado por los sujetos de estudio.

La información recopilada se analizó a partir de la distribución de frecuencia de los porcentajes de cada afirmación, buscando diferencias significativas según nivel de conciencia manifestado por los sujetos encuestados. Dado que los datos son de tipo ordinales se utilizó la prueba Kruskal-Wallis en la cual $\mathrm{H}_{0}$ (hipótesis nula) nos señala que no hay diferencias entre los grupos y $\mathrm{H}_{1}$ (hipótesis alternativa) nos indica que al menos uno de los grupos es diferente. En esta investigación se rechazará la $\mathrm{H}_{0}$ cuando el valor de $p$ obtenido sea menor o igual a 0.05 .

La prueba Kruskal-Wallis corresponde a un método no paramétrico que no debe ajustarse a ninguna distribución, es decir, no requiere que los datos presentan distribución normal o alguna otra distribución de forma específica. Por tanto, puede aplicarse incluso aunque no se cumplan las condiciones de validez paramétricas. Además, las pruebas no paramétricas son más robustas que las paramétricas. En otras palabras, son válidas en un rango más amplio de situaciones, dado que exigen menos condiciones que respalden dicha validez, por lo que se ajusta a las necesidades de esta investigación en función de la gran cantidad y diversidad de datos recolectados y al uso de una escala tipo Likert con intensidad creciente con el fin de establecer una escala de puntajes (Villaroel del Pino, 2018). En cuando al uso de software de análisis estadísticos, en esta investigación se utilizó el programa Stata versión 14 en español.

\section{RESULTADOS Y DISCUSIÓN}

Los datos obtenidos fueron compilados en tres tablas: en la tabla 1 se sintetiza la distribución de las respuestas en relación con las afirmaciones respecto a la "Conciencia de la actividad" señalando los porcentajes según nivel en la escala Likert. En las tablas 2 y 3 se describen la distribución de las respuestas respecto al uso de herramientas para la generación de contenidos y a la creación de información, en estas dos tablas los resultados fueron agrupados según el nivel de conciencia de actividad, señalando el valor-p correspondiente para identificar si existe o no diferencias significativas entre niveles.

En las tablas 1, 2, y 3 se utilizará la siguiente nomenclatura $\left({ }^{a}\right): S / C S=$ Siempre o Casi siempre; $A M=A$ menudo; $\mathrm{AV}=\mathrm{A}$ veces; $\mathrm{PV}=$ Pocas veces; $\mathrm{CN} / \mathrm{N}=$ Casi nunca/Nunca y $\mathrm{UN} / \mathrm{NA}=\mathrm{No}$ usa/aplica. En las tablas 2 y 3 , además, se utilizará la nomenclatura ${ }^{(b)} A=$ Alta conciencia de la actividad; $M=$ mediana conciencia de la actividad; $\mathrm{B}=$ Baja conciencia de la actividad. En todos los casos se considerará que existe significancia cuando $\mathrm{p}<0.05$, lo cual estará indicado por el siguiente símbolo $\left(^{*}\right)$.

Tabla 1: Distribución de las respuestas según afirmación asociada a la conciencia de la actividad.

\begin{tabular}{|l|c|c|c|c|c|c|}
\hline $\begin{array}{l}\text { Me siento más motivado o me activa a participar más } \\
\text { cuando conozco... }\end{array}$ & \multicolumn{5}{|c|}{ Distribución porcentual(a) } \\
\cline { 2 - 6 } & $S / C S$ & $A M$ & $A V$ & $P V$ & CN/N & NU/NA \\
\hline $\begin{array}{l}\text { Número de seguidores/amigos que tengo en las redes } \\
\text { sociales. }\end{array}$ & $19.65 \%$ & $15.52 \%$ & $17.49 \%$ & $17.49 \%$ & $17.88 \%$ & $11.98 \%$ \\
\hline $\begin{array}{l}\text { Cantidad de retweets/likes/me gusta que registran mis } \\
\text { publicaciones. }\end{array}$ & $15.72 \%$ & $13.95 \%$ & $16.11 \%$ & $14.54 \%$ & $15.32 \%$ & $24.36 \%$ \\
\hline $\begin{array}{l}\text { Cantidad de personas que asistirán a eventos creados } \\
\text { por mí. }\end{array}$ & $9.63 \%$ & $12.18 \%$ & $12.38 \%$ & $11.59 \%$ & $16.70 \%$ & $37.52 \%$ \\
\hline $\begin{array}{l}\text { Cantidad de personas que aceptan tus invitaciones a } \\
\text { grupos creados por mí. }\end{array}$ & $8.25 \%$ & $10.61 \%$ & $11.00 \%$ & $12.97 \%$ & $19.25 \%$ & $37.92 \%$ \\
\hline $\begin{array}{l}\text { Cantidad de grupos en los que participo. } \\
\text { Cantidad de fotografías que he publicado. }\end{array}$ & $11.39 \%$ & $13.75 \%$ & $12.57 \%$ & $19.06 \%$ & $23.38 \%$ & $19.84 \%$ \\
\hline Las métricas de mis publicaciones (estadísticas). & $9.43 \%$ & $11.98 \%$ & $12.97 \%$ & $17.09 \%$ & $19.06 \%$ & $29.47 \%$ \\
\hline
\end{tabular}

Al asignar puntaje a cada uno de los niveles de la escala Likert que se observan en la tabla 1 y siguiendo los criterios para la establecer los niveles de conciencia, determinó que el $10.81 \%$ de los sujetos obtuvo un puntaje entre 42 y 22 puntos, clasificándolos como de "Alta conciencia de la actividad". Con un puntaje entre 21 y 15 , el $47.35 \%$ de los sujetos fue clasificado como de "Mediana conciencia de la actividad". Finalmente, con un puntaje entre 14 y 7 el $41.85 \%$ de los sujetos fue clasificado como de "Baja conciencia de la actividad".

En la tabla 1 se observa que, dentro de los aspectos consultados, la mayor conciencia se encuentra en relación con la "Cantidad de fotografías que han publicado", con un acumulado de un $36.55 \%$ entre las opciones S/CS y AM, seguido por el Conocimiento del "Número de seguidores" entre las mismas opciones, con un $35.17 \%$ y en tercer lugar, la "Cantidad de retweets/likes/me gusta" que registran sus publicaciones, 
con un $29.67 \%$, considerando las mismas dos opciones de respuesta. También en la misma tabla 1 se puede evidenciar que la información sobre la que tienen menos conciencia, es con respecto a la cantidad de personas que aceptan sus invitaciones a los eventos que ellos crean, que acumula un $57.17 \%$, entre las opciones $\mathrm{CN} / \mathrm{N}$ y NU/NA, seguido con el conocimiento de las personas que asistirán a dichos eventos con un $54.22 \%$ y en tercer lugar se encuentra el conocimiento de las métricas (estadística) de sus publicaciones con un acumulado de un $48.53 \%$ en las opciones $\mathrm{CN} / \mathrm{N}$ y NU/NA.

Tabla 2: Distribución de las respuestas según afirmación asociada al uso de herramientas para la creación de la información por nivel de conciencia de la actividad.

\begin{tabular}{|c|c|c|c|c|c|c|c|c|}
\hline \multirow{2}{*}{$\begin{array}{l}\text { Cuando quiero generar nueva } \\
\text { información para publicarla en la } \\
\text { red: }\end{array}$} & \multirow{2}{*}{$\begin{array}{l}\text { Nivell } \\
\text { b) }\end{array}$} & \multicolumn{6}{|c|}{ Distribución de frecuencia y porcentual(a) } & \multirow[b]{2}{*}{ Valor-p } \\
\hline & & S/CS & $A M$ & $A V$ & $P V$ & $\mathrm{CN} / \mathrm{N}$ & NU/NA & \\
\hline \multirow[t]{3}{*}{ No sé hacerlo } & A & $20.00 \%$ & $25.45 \%$ & $25.45 \%$ & $9.09 \%$ & $10.91 \%$ & $9.09 \%$ & \multirow{3}{*}{$0.0050^{(*)}$} \\
\hline & $\mathrm{M}$ & $14.55 \%$ & $14.08 \%$ & $15.02 \%$ & $12.68 \%$ & $17.37 \%$ & $26.29 \%$ & \\
\hline & $B$ & $21.58 \%$ & $14.11 \%$ & $12.86 \%$ & $10.79 \%$ & $17.84 \%$ & $22.82 \%$ & \\
\hline \multirow{3}{*}{$\begin{array}{l}\text { Publico la información en una red } \\
\text { social que utilizo habitualmente. }\end{array}$} & A & $18.18 \%$ & $27.27 \%$ & $30.91 \%$ & $7.27 \%$ & $0.00 \$$ & $16.36 \%$ & \multirow[t]{3}{*}{$0.0001^{(*)}$} \\
\hline & $M$ & $32.86 \%$ & $25.82 \%$ & $14.55 \%$ & $8.92 \%$ & $7.51 \%$ & $10.33 \%$ & \\
\hline & $\mathrm{B}$ & $14.94 \%$ & $19.92 \%$ & $17.01 \%$ & $15.35 \%$ & $11.20 \%$ & $21.58 \%$ & \\
\hline \multirow{3}{*}{$\begin{array}{l}\text { Utilizo una herramienta específica } \\
\text { (tipo blog) }\end{array}$} & A & $5.45 \%$ & $16.36 \%$ & $29.09 \%$ & $10.91 \%$ & $7.27 \%$ & $30.91 \%$ & \multirow[t]{3}{*}{$0.0004^{(*)}$} \\
\hline & $\mathrm{M}$ & $7.98 \%$ & $12.68 \%$ & $10.80 \%$ & $16.90 \%$ & $21.90 \%$ & $30.05 \%$ & \\
\hline & $\mathrm{B}$ & $3.73 \%$ & $6.64 \%$ & $12.45 \%$ & $14.11 \%$ & $19.92 \%$ & $43.15 \%$ & \\
\hline \multirow{3}{*}{$\begin{array}{l}\text { Según el tipo de información utilizo } \\
\text { una herramienta u otra (blog, red } \\
\text { social, Google Sites, etc.) }\end{array}$} & $A$ & $9.09 \%$ & $21.82 \%$ & $20.20 \%$ & $18.18 \%$ & $7.27 \%$ & $23.64 \%$ & \multirow[t]{3}{*}{$0.0001^{(*)}$} \\
\hline & $M$ & $15.96 \%$ & $13.62 \%$ & $18.78 \%$ & $11.27 \%$ & $14.55 \%$ & $25.82 \%$ & \\
\hline & B & $6.22 \%$ & $10.37 \%$ & $14.52 \%$ & $13.28 \%$ & $18.67 \%$ & $36.93 \%$ & \\
\hline
\end{tabular}

En tabla 2 podemos ver que el uso de herramientas específicas y el uso de una herramienta según tipo de información es más frecuente entre individuos con "Alta conciencia de su actividad" con un $21.81 \%$ y un $30.91 \%$ respectivamente al sumar los porcentajes de las opciones S/CS y AM. Estos porcentajes son muy cercanos a los que presenta el grupo que posee una "Mediana conciencia de la actividad", donde el uso de herramientas específicas llega a un $20.66 \%$ (S/CS-AM) y el uso de herramientas según tipo de información llega a un $29.58 \%$. El grupo con "Baja conciencia de la actividad" llega a un $10.37 \%$ (S/CS-AM) en cuanto al uso de herramientas específicas, y a un $16.59 \%$ (S/CS-AM) respecto al uso de herramientas según tipo de información.

En la misma tabla 2 se indica que la publicación de información en redes sociales de uso habitual es mucho más frecuente entre personas con una mediana conciencia de su actividad con un $58.68 \%$ (S/CS-AM). Los sujetos que presentan una alta conciencia de la actividad llegan a un $45.45 \%$ (S/CS-AM y el grupo que presenta una baja conciencia de la actividad llega a un 34.86\% (S/CS-AM). En cuanto a la declaración de no saber cómo generar nueva información, en el grupo con alto nivel de conciencia solo un $20 \%$ respondió en las opciones CN/N-NU/NA que daría a entender que sí sabrían hacerlo. En los grupos con mediana conciencia y baja conciencia, los porcentajes aumentan a un $43.66 \%$ (CN/N-UN/NA) y a un $40.66 \%$ (CN/N-UN/NA) respectivamente.

Por otro lado, en la tabla 3 se señala que la publicación de contenido de tipo icónico como las fotografías es más común en quienes tienen una mediana conciencia de su actividad con un $66,67 \%$ (S/CS-AM), quedando los grupos con una alta conciencia de la actividad y baja conciencia de la actividad en condiciones muy similares de frecuencia, con un $49.09 \%$ (S/CS-AM) y un $49.38 \%$ (S/CS-AM) respectivamente.

En cuanto a la publicación de contenidos digitales del tipo multimedia e hipermedia (ver tabla 3), estas son más frecuentes en quienes tienen una alta conciencia de su actividad coincidiendo con un $20 \%$, en ambos casos al sumar las preferencias S/CS y AM, siendo ambos el cuarto tipo de contenidos más común en cuanto a producción dentro de este grupo. En el caso de las personas con una mediana conciencia de la actividad, la creación de contenidos multimedia llega a un $19.72 \%$ entre las opciones S/CS-AM, siendo también el cuarto tipo de contenido más comúnmente producido, pero en cuanto a la creación de contenidos de tipo hipermedia el porcentaje baja a un $12.67 \%$ (S/CS- AM), posicionándolo en el sexto lugar entre las opciones de contenido digital producido y compartido por los estudiantes. En cuando al grupo de baja conciencia, la producción de multimedia es de un $8.71 \%$ (S/SC-AM) y la de hipermedia es de un $6.63 \%$ (S/CS-AM).

La producción de videos que se señala en la tabla 3 se sitúa como el segundo tipo de contenidos más común en los tres grupos, llegando a un $28,64 \%$ (S/CS-AM) en el caso de quienes presentaban una mediana conciencia de la actividad. En el caso de los sujetos de alta conciencia de la actividad este porcentaje llega a 
un $27,27 \%$ (S/CS-AM). En el grupo de baja conciencia de la actividad el porcentaje llega a un $17.84 \%$ (S/CSAM). El uso de textos es la tercera opción más común para quienes tiene una mediana conciencia de su actividad con un $25,83 \%$ (S/CS-AM), estando eso sí muy por debajo que el uso de contenidos de tipo icónico. El uso de textos en personas con alta conciencia de su actividad llegó a un $23.63 \%$ (S/CS-AM), siendo también la tercera opción con mayor preferencia. Es interesante observar que de la tabla 2 se extrapola que un $20.00 \%$ de quienes posee una alta conciencia de la actividad sabrían cómo generar información y, al mismo tiempo, un $20.00 \%$ de este grupo declara producir contenidos de tipo multimedia e hipermedia.

Tabla 3: Distribución de las respuestas según afirmación asociada al tipo de contenido para la creación de la información por nivel de conciencia de la actividad

\begin{tabular}{|c|c|c|c|c|c|c|c|c|}
\hline \multirow{2}{*}{$\begin{array}{l}\text { Los contenidos digitales que } \\
\text { produzco para compartir en red } \\
\text { son: }\end{array}$} & \multirow[b]{2}{*}{ Nivel ${ }^{(b)}$} & \multicolumn{6}{|c|}{ Distribución de frecuencia y porcentual(a) } & \multirow[b]{2}{*}{ Valor-p } \\
\hline & & $\mathrm{S} / \mathrm{CS}$ & AM & AV & PV & $\mathrm{CN} / \mathrm{N}$ & UN/NA & \\
\hline \multirow[t]{3}{*}{ Texto (wikis, blog) } & A & $5.45 \%$ & $18.18 \%$ & $29.09 \%$ & $12.73 \%$ & $10.91 \%$ & $23.64 \%$ & \multirow[t]{3}{*}{$0.0008^{*}$} \\
\hline & M & $13.15 \%$ & $12.68 \%$ & $19.72 \%$ & $15.02 \%$ & $12.21 \%$ & $27.23 \%$ & \\
\hline & B & $4.98 \%$ & $11.62 \%$ & $16.18 \%$ & $11.20 \%$ & $18.67 \%$ & $37.34 \%$ & \\
\hline \multirow{3}{*}{$\begin{array}{l}\text { Icónicas (fotografías o } \\
\text { imágenes en Flickr, Instagram, } \\
\text { etc.) }\end{array}$} & $A$ & $29.09 \%$ & $20.00 \%$ & $23.64 \%$ & $3.64 \%$ & $10.91 \%$ & $12.73 \%$ & \multirow[t]{3}{*}{$0.0001^{(*)}$} \\
\hline & M & $38.03 \%$ & $28.64 \%$ & $14.55 \%$ & $6.10 \%$ & $2.82 \%$ & $9.86 \%$ & \\
\hline & B & $23.65 \%$ & $25.73 \%$ & $15.77 \%$ & $9.96 \%$ & $6.22 \%$ & $18.67 \%$ & \\
\hline \multirow[t]{3}{*}{ Video (YouTube, Vimeo, etc.). } & $A$ & $7.27 \%$ & $20.00 \%$ & $25.45 \%$ & $10.91 \%$ & $16.36 \%$ & $20.00 \%$ & \multirow[t]{3}{*}{$0.0002^{(*)}$} \\
\hline & M & $12.68 \%$ & $15.96 \%$ & $18.31 \%$ & $12.68 \%$ & $15.49 \%$ & $24.88 \%$ & \\
\hline & $\mathrm{B}$ & $8.30 \%$ & $9.54 \%$ & $11.20 \%$ & $13.69 \%$ & $21.99 \%$ & $35.27 \%$ & \\
\hline \multirow[t]{3}{*}{ Audio (podcast). } & A & $10.91 \%$ & $7.27 \%$ & $16.36 \%$ & $14.55 \%$ & $20.00 \%$ & $30.91 \%$ & \multirow[t]{3}{*}{$0.0009^{(*)}$} \\
\hline & M & $7.51 \%$ & $11.74 \%$ & $9.39 \%$ & $15.02 \%$ & $19.25 \%$ & $37.09 \%$ & \\
\hline & B & $4.15 \%$ & $5.39 \%$ & $7.88 \%$ & $10.79 \%$ & $24.90 \%$ & $46.89 \%$ & \\
\hline \multirow{3}{*}{$\begin{array}{l}\text { Multimedia (SlideShare, } \\
\text { Animoto, etc.) }\end{array}$} & A & $3.64 \%$ & $16.36 \%$ & $16.36 \%$ & $12.73 \%$ & $21.82 \%$ & $29.09 \%$ & \multirow[t]{3}{*}{$0.0001^{(*)}$} \\
\hline & M & $6.57 \%$ & $13.15 \%$ & $13.62 \%$ & $15.96 \%$ & $17.84 \%$ & $32.86 \%$ & \\
\hline & B & $2.90 \%$ & $5.81 \%$ & $9.96 \%$ & $13.28 \%$ & $20.33 \%$ & $47.72 \%$ & \\
\hline \multirow{3}{*}{$\begin{array}{l}\text { Hipermedia (eXeLearning, } \\
\text { página web en Wix u otras) }\end{array}$} & A & $12.73 \%$ & $7.27 \%$ & $14.55 \%$ & $16.36 \%$ & $18.18 \%$ & $30.91 \%$ & \multirow[t]{3}{*}{$0.0001^{(*)}$} \\
\hline & M & $4.69 \%$ & $7.98 \%$ & $8.92 \%$ & $12.68 \%$ & $17.37 \%$ & $48.36 \%$ & \\
\hline & $B$ & $2.90 \%$ & $3.73 \%$ & $5.81 \%$ & $7.88 \%$ & $25.31 \%$ & $54.36 \%$ & \\
\hline
\end{tabular}

Si bien esta investigación es un estudio de caso, ya que los sujetos participantes fueron del tipo voluntarios y no elegidos al azar, los resultados obtenidos en rigor son válidos solo para la muestra. No obstante, si consideramos los siguientes aspectos, podemos afirmar que dichos resultados pueden ser considerados como una buena aproximación a los que se obtendría en un estudio aleatorio: 1. El tamaño de la muestra fue grande (509 sujetos), tomando en cuenta que el tamaño muestral calculado para una muestra aleatoria con una población infinita (muy grande) sería de 384 sujetos. 2. Ambos instrumentos utilizados fueron validados por grupos de expertos y, además, se realizó una validación psicométrica en población chilena. 3. Se cubrió el $38.19 \%$ de las universidades chilenas (21/55). 4. Las universidades participantes estaban localizadas tanto en la zona norte, centro y sur de Chile, lo que asegura una representatividad geográfica.

Un primer aspecto para discutir tiene relación con el hecho de que cuando pasamos de un nivel de conciencia mediano a bajo disminuye, considerablemente, la producción de contenido en línea; centrándose esta sobre todo en los contenidos de tipo icónico. En redes sociales los contenidos visuales presentan una producción mayoritaria por sobre los de audios, textos, multimedia e hipermedia, no utilizando herramientas específicas para su publicación. En tanto, cuando se llega a un nivel de mediana conciencia la producción de contenidos aumenta, centrándose, principalmente, en la producción icónica, pero con un incremento considerable en la producción de videos, audios, textos, multimedia e hipermedia; con el respectivo incremento en el uso de herramientas específicas. Lo cual nos hace inferir que la conciencia de la actividad se asociaría con el tipo de productividad y la utilización de herramientas específicas para su generación.

Una posible explicación respecto de este hallazgo dice relación con que los universitarios chilenos, al igual que los ecuatorianos, ven en el uso de celulares su principal herramienta tecnológica (Hernández et al., 2021; 
Basantes et al., 2017). Por tanto, la producción que realizan está en directa sintonía con las herramientas que dicho artefacto tecnológico les provee y, si consideramos que, la identidad se confirma a través de procesos de reconocimiento social donde la experiencia de nuestro yo -en interacción con otros- se va a ir nutriendo y adquiriendo conciencia en esta interrelación. La experiencia será, más o menos favorable, en directa relación con las competencias digitales que se tienen para generar actividad en la Red. En tanto, cuando pasamos al nivel de alta conciencia de la actividad nos encontramos con el hecho de que el $45.45 \%$ de los sujetos declaran no saber cómo publicar información que quieren generar y que solo un $20 \%$ declara saber cómo hacerlo. Esto es consistente con el porcentaje de productores de multimedia e hipermedia en esta investigación.

Ahora bien, ¿cómo se explica el hecho de que a pesar de ser altamente conscientes de su actividad no generan contenidos e incluso declaran no saber cómo hacerlo? Para poder dar una respuesta, debemos entender que los participantes en la Red pueden ser clasificados en dos grandes grupos: los observadores (lurkers) y los publicadores (posters), los primeros leen y observan sin hacer su propia retribución mientras que los segundos comparten información en línea más allá del like, pero no necesariamente llegar al nivel de un prosumidor (Nguyen, 2020). Por otra parte, los observadores pueden, incluso, llegar a convertirse en acosadores o stalkers (Howard et al., 2017). Segmento que suelen representar, en algunos casos, hasta el $90 \%$ de total de participantes en una comunidad o red; conducta común y que no representa algo negativo, salvo en situaciones puntuales cuando comienzan a existir conductas de observación proclives al acoso (Cranefield et al., 2015).

Nuestra investigación coincide con los hallazgos del estudio realizado en estudiantes turcos, donde sus PLE son entornos útiles en el fácil acceso a contenidos e interacción con otros usuarios; permitiendo el aprendizaje, pero no están centrados en gestionar o construir más aprendizaje, tornándose en actores más bien pasivos (Şahin et al., 2016). Ajustándose, la población en estudio, más a observadores que a publicadores y prosumidores.

Si bien los observadores no participan activamente en la publicación, igualmente son parte interesada en el proceso de intercambio de conocimientos. Desde una perspectiva de intercambio de información, el conocimiento es el valor que atrae a los miembros a las comunidades en línea, es decir, los observadores son atraídos por los publicadores. Por tanto, siempre su número será mayor, pero para poder ser un buen observador es importante una actitud activa y consciente que les permita acceder fácil y rápidamente a la información que se busca (Nguyen, 2020). De acuerdo con lo anterior, nuestros resultados son coincidentes con las conclusiones de la revisión realizada por Nguyen (2020), a partir del análisis de 100 investigaciones aplicadas en países pertenecientes a Europa, Asia y Norteamérica, como también con la investigación española realizada por Torres Kompen et al. (2019) que devela que las herramientas y estrategias que los estudiantes escogen para conformar su PLE son un punto de partida que varía con el conocimiento y conciencia que se tenga respecto del objetivo a alcanzar y, por ende, se transitaría a adoptar un enfoque más de prosumidor que de consumidor cuando este proceso se hace más consciente.

Un segundo aspecto para discutir dice relación con los tipos de PLE, en donde las herramientas tecnológicas seleccionadas por los usuarios van más allá del proceso de aprendizaje que involucra las esferas formales e informales de acceso a la información, sino también, a las esferas personales y profesionales involucradas en sus redes personales de aprendizaje. Acá entran en acción todas aquellas herramientas de la Web 4.0 que le conectan con su mundo académico, pero también profesional. El estudio de Salam Abdallah (2018) evidencia que el intercambio de información, los códigos y el tipo de lenguaje compartido en las redes no solo dan cuenta sobre la identidad de los usuarios, sino también, de su reputación ligada intrínsecamente a la producción y a las publicaciones que este realice. En este escenario la participación, aunque sea una emulación o copia a otros con una alta reputación, es legitimada por la comunidad como una forma de fortalecimiento del grupo en sí mismo. Instando a que, en la medida, que se tiene pertenencia y conciencia de ello aumenta la participación y el compromiso social, conllevando la generación de mejores contenidos.

Si bien nuestra investigación se aleja de los resultados evidenciados en la población árabe analizada, al carecer de un escenario proclive a la producción de contenidos valiosos -o al menos que implican un tratamiento tecnológico más elaborado por medio de aplicaciones web- sí se puede corroborar que la producción tiene estrecha relación con el grado de conciencia de la actividad desplegada y manifiesta por el usuario (Abdallah, 2018). Es decir, que los estudiantes universitarios chilenos al mostrar una baja conciencia de la actividad social donde las métricas, la cantidad de personas que asistirán a eventos o aceptaron invitaciones a grupos creados por ellos es baja, de igual forma, su producción es baja y ligada a competencias digitales básicas, centrándose en producciones icónicas (fotografías) y de texto, principalmente. De allí que un número de contenidos digitales del tipo multimedia e hipermedia son más frecuentes en quienes tienen una alta conciencia de su actividad y que no superan el $20 \%$ de la población en estudio. Lo que está unido a que este tipo de producciones de información requiere tanto del manejo de herramientas digitales, como también del despliegue de tareas de pensamiento de orden superior. Implica que los estudiantes procesen la información aprendida y sean capaces de diseñar, formular y crear nueva información (Reyna et al., 2017). 
Cabe preguntarnos si el uso de herramientas para la creación de contenidos que sean más complejos que uno o dos clics (como lo es la generación de imágenes y su distribución en redes sociales) no es una habilidad que surja de manera natural y espontánea o como lo mencionan Reyna et al., (2017) se puede colegir que estas producciones están denotando que los universitarios no están logrando tareas de pensamiento de orden superior. Independiente de la respuesta, a esta y otras muchas incógnitas que pueden nacer de la información entregada por esta investigación, debemos tener presente que, para lograr una transición efectiva en habilidades digitales, es necesario, considerar tanto las características constitutivas de la identidad digital de los jóvenes universitarios como sus PLE. Dado que denotan deficiencias en el uso de las herramientas digitales y producción de artefactos tecnológicos de cuya acción con alta, media o baja conciencia de actividad quedaran huellas registradas sempiternamente en la Web. Asimismo, como docentes, es necesario que se resignifiquen los espacios de aprendizaje, echando un vistazo a lo que realmente utiliza el estudiante en su día a día. Sin limitarse a áreas unidimensionales: presencial versus digital, público versus privado y formal versus informal. Reconsiderando la forma en que se integra y piensa el proceso de enseñanza-aprendizaje con las herramientas digitales y abriendo la puerta a crear nuevas oportunidades de aprendizaje con herramientas tecnológicas "no convencionales".

A la luz de los hallazgos de la investigación y, en consonancia con el escenario educativo puesto al descubierto por la pandemia de SARS-CoV-2 que comenzó en 2019, los estudiantes pertenecientes a la generación $Z$ coexisten con tecnologías 3.0 y 4.0 y su identidad digital será reflejo del manejo de herramientas digitales unidas al grado de conciencia que se tenga de dicha actividad. Cabe destacar que en esta tarea debieran ser guiados por los docentes, pero los chilenos y latinoamericanos, en general, no cuentan con las habilidades y competencias digitales necesarias para llevar a cabo procesos de enseñanza y aprendizaje en entornos digitales (Kumar et al., 2018). Chile, al igual que otros países de América Latina, recién está sentando las bases necesarias para pasar del e-learning 2.0 al d-Learning o aprendizaje digital. Debido a esto, durante la pandemia, en lugar de hablar de un proceso de formación e-Learning, la comunidad académica prefirió hablar de educación remota o educación remota de emergencia apoyada con tecnología.

Lo anterior invita a replantearnos respecto de los usuarios y futuros profesionales que están egresando y que, prontamente, se insertarán en el mundo laboral, cuyas habilidades de alfabetización mediática necesarias para producir artefactos digitales son precarias (Brandtzaeg, 2020), pero también insta a que comprendamos los fenómenos de las tecnologías digitales desde el individuo y la sociedad. Las oportunidades y/o desigualdades estarán dadas por la capacidad que tengan las personas como usuarios para adaptarse a una sociedad de acelerados cambios, en la que los medios de comunicación y la cultura digital se diversifican y adquieren un papel preponderante. De modo que, con cada selfie, like, transmisión en vivo, post u otra producción compartida en la Web se aportará a la construcción de la identidad mundial del ciberespacio y, por ende, a la identidad digital de cada uno de los usuarios (Reyna et al., 2017; Abdallah, 2018; Çöteli, 2019). Finalmente, la actuación de dicha identidad se verá explicitada en cada texto y su respectivo vocabulario, así como en el contenido y la redacción de cada publicación, la que -en definitiva- será fuente de acceso y aceptación o rechazo del usuario a las redes sociales de aprendizaje en general, como de entornos profesionales, acompañándole una reputación digital favorable o desfavorable, según sea el caso.

\section{CONCLUSIONES}

De acuerdo con los resultados de este estudio y de su discusión, de la comparación con resultados de otros autores y del detallado análisis presentado, se pueden extraer las siguientes conclusiones:

1) Una menor conciencia de la actividad implica una menor producción de contenidos y así mismo una menor utilización de herramientas específicas para su generación.

2) Una mayor conciencia de la actividad podría implicar tanto la presencia de un grupo de personas altamente productiva que utiliza herramientas específicas para su producción o un grupo de observadores altamente activos que no generan contenidos, sino que se limitan a su consumo constante, lo que los lleva ser altamente conscientes de su actividad de manera de poder tener un alto acceso a la información de su interés y darle seguimiento.

3) Los PLE de los universitarios reflejan no solo sus competencias digitales, sino también, su grado de conciencia de actividad por lo que la producción de artefactos tecnológicos y comunicación en medios digitales que desplieguen serán los que configurarán su identidad digital.

\section{REFERENCIAS}

Abdallah, S., Digital Identity and Reputation in a Virtual Practice Network-Insights Using Linguistic

Features, http://dx.doi.org/10.4018/ljeC.2018100101, International Journal of e-Collaboration (IJeC), 14(4), 1-23 (2018). 
Barroso Osuna, J., y Cabero Almenara, J., La Utilización del Juicio de Experto para la Evaluación de TIC: El Coeficiente de Competencia Experta, http://dx.doi.org/10.13042/brp.2013.65202, Bordón, 65(2), 25-38 (2013).

Basantes, A., Naranjo, M., Gallegos, M., y Benítez, N., Los Dispositivos Móviles en el Proceso de Aprendizaje de la Facultad de Educación Ciencia y Tecnología de la Universidad Técnica del Norte de Ecuador, http://dx.doi.org/10.4067/S0718-50062017000200009, Formación Universitaria, 10(2), 79-88 (2017).

Bauman, Z., Emancipación, Modernidad líquida, 1a Reimpresión, Fondo de Cultura Económica, 21-40, México, D.F., México (2015).

Belli, S., y De Eugenio, G., Emotional Practices and Processes of Subjectivation in Digital Society: The Case of Online Suicides, http://dx.doi.org/10.5565/rev/qpsicologia.1181, Quaderns de Psicología, 16(2), 57-72 (2014).

Brandtzaeg, P., y Chaparro-Domínguez, M. Á., From Youthful Experimentation to Professional Identity: Understanding Identity Transitions in Social Media, http://dx.doi.org/10.1177/1103308819834386, Young, 28(2), 157-174 (2020).

Castañeda, L., y Camacho, M., Desvelando Nuestra Identidad Digital, https://doi.org/10.3145/epi.2012.jul.04, El Profesional de la Información, 21(4), 354-360 (2012).

Çöteli, S., The Impact of New Media on The Forms of Culture: Digital Identity and Digital Culture, http://dx.doi.org/10.29333/ojcmt/5765, Online Journal of Communication and Media Technologies, 9(2) (2019).

Cranefield, J., Yoong, P., y Huff, S. L., Rethinking Lurking: Invisible Leading and Following in a Knowledge Transfer Ecosystem, Journal of the Association for Information Systems, 16(4), 213 (2015).

Dabbagh, N., y Castañeda, L. The PLE as a Framework for Developing Agency in Lifelong Learning. http://dx.doi.org/10.1007/s11423-020-09831-z, Educational Technology Research and Development, 68, 3041-3055 (2020).

Esteve-González, V.; González, J.; Gisbert, M., y Cela-Ranilla, J., La Presencia Social en Entornos Virtuales 3D: Reflexiones a Partir de una Experiencia en la Universidad, http://dx.doi.org/10.12795/pixelbit.2017.i50.09, Pixel-Bit, 50, 137-146 (2017).

Feher, K., Digital Identity and the Online Self: Footprint Strategies-An Exploratory and Comparative Research Study, http://dx.doi.org/10.1177/0165551519879702, Journal of Information Science, 47(2),192-205 (2019).

Georgalou, M., I Make the Rules on My Wall': Privacy and Identity Management Practices on Facebook, doi:10.1177/1750481315600304, Discourse Commun, 10, 40-64 (2016).

Georges, F., A l'image de l'Homme: cyborgs, avatars, identités numériques, http://dx.doi.org/10.3917/tdm.018.0136, Le Temps des Médias,18(1), 136-147 (2012).

Guodong, W., y Xinghua, S., Building a Personal Learning Environment with IFTTT Service, http://dx.doi.org/10.3923/jse.2016.431.436, Journal of Software Engineering, 10, 431-436 (2016).

Hernández, M.; Perez, A., y Roco, A., Identidad digital y conectividad: conocimiento y actitudes en estudiantes universitarios chilenos, http://dx.doi.org/10.4067/S0718-50062021000100147, Formación Universitaria, 14(1), 147-156 (2021).

Howard, D. E., Debnam, K. J., y Strausser, A., "I'm a Stalker and Proud of It": Adolescent Girls' Perceptions of the Mixed Utilities Associated With Internet and Social Networking Use in Their Dating Relationships, http://dx.doi.org/10.1177/0044118X17716948, Youth \& Society, 51(6) 773-792 (2017).

Huang, J., Kumar, S., y Hu, C., Gender Differences in Motivations for Identity Reconstruction on Social Network Sites, http://10.0.4.56/10447318.2017.1383061, International Journal of Human-Computer Interaction, 34(7), 591-602 (2018).

Kumar S., Wotto M., y Bélanger P., E-learning, M-learning and D-learning: Conceptual definition and comparative analysis, https://journals.sagepub.com/doi/abs/10.1177/2042753018785180, E-Learning and Digital Media, 15(4), 191216 (2018).

Lange, J., Platform Stabilization: An Autoethnographic Exploration of The Multiple Relations and Role of Data Behind the Interface of Online Tutoring Software, http://dx.doi.org/10.1080/17508487.2020.1786708, Critical Studies in Education, 115 (2020).

Nguyen, T. M., Four-Dimensional Model: A Literature Review on Reasons Behind Lurking Behavior, https://doi.org/10.1108/VJIKMS-10-2019-0168, VINE Journal of Information and Knowledge Management Systems, $51(2), 392-317$ (2020).

Prendes-Espinosa, M. P., Castañeda-Quintero, L., y otros cuatro autores, Validación de un cuestionario sobre hábitos de trabajo y aprendizaje para futuros profesionales: Explorar los Entornos Personales de Aprendizaje, https://dx.doi.org/10.7203/relieve.22.2.7228, Relieve, 22(2), 1-46 (2016).

Rejón-Guardia, F., Polo-Peña, A. I., y Maraver-Tarifa, G., The Acceptance of a Personal Learning Environment Based on Google Apps: The Role of Subjective Norms and Social Image, http://dx.doi.org/10.1007/s12528-019-09206-1, Journal of Computing in Higher Education, 32(2), 203-233 (2020).

Reyna, J., Hanham, J., y Meier, P., Taxonomy of Digital Media Types for Learner-Generated Digital Media Assignments, http://dx.doi.org/10.1177/2042753017752973, E-Learning and Digital Media, 14(6), 309-322 (2017). 
Şahin, S., y Uluyol, Ç., Preservice Teachers' Perception and Use of Personal Learning Environments (PLEs), http://dx.doi.org/10.19173/irrodlv17i2.2284, The International Review of Research in Open and Distributed Learning, 17(2), 141-161 (2016).

Sibilia, P., Introducción, El Hombre Postorgánico. Cuerpo, Subjetividad y Tecnologías Digitales, 2ª Ed., Fondo de Cultura Económica, 11-15, Buenos Aires, Argentina (2010).

Torres Kompen, R., Edirisingha, P., y otros tres autores, Personal Learning Environments Based on Web 2.0 Services in Higher Education, https://dx.doi.org/10.1016/j.tele.2018.10.003, Telematics and Informatics, 38, 194-206 (2019).

Villaroel, L., Test de hipótesis y asociación de variables, Métodos Bioestadísticos, 2ª Ed., Ediciones UC, 135-206, Santiago, Chile (2018).

Wiens, B. I., y MacDonald, S., Feminist Futures: \#MeToo's Possibilities as Poiesis, Techné, and Pharmakon, http://dx.doi.org/10.1080/14680777.2020.1770312, Feminist Media Studies, 1-17 (2020).

Winocur, R., La Intimidad de Los Jóvenes en Las Redes Sociales: Transformaciones en el Espacio Público y Privado, Telos: Cuadernos de Comunicación e Innovación, 91, 79-88 (2012). 